\title{
EMPRÉSTIMO LEXICAL, CONCEPTUALIZAÇÃO E VARIAÇÃO: PARA A ABORDAGEM SOCIOCOGNITIVA E SOCIOLETOMÉTRICA DOS ESTRANGEIRISMOS NO PORTUGUÊS
}

\author{
AUGUSTO SOARES DA SILVA
}

RESUMO

This paper first advocates an onomasiological, concept-based and socio-cognitive approach to lexical borrowing, expanding the current loanword research from lexical items towards concepts. Second, it presents a corpusbased and concept-based sociolectometrical study on differences in the use of loanwords in European Portuguese and Brazilian Portuguese and their impact on diachronic lexical variation between the two national varieties. In the first part, the main topics and contributions of the Cognitive Sociolinguistic perspective on borrowability, and concept-based sociolectometrical methods of measuring variation in the success of loanwords are highlighted. In the second part, English and French loanwords in the field of football and clothing terminologies are analyzed through possible receptor Portuguese equivalents and advanced corpus-based sociolectometrical measures, such as featural measures (calculating the proportion of terms possessing a special feature) and uniformity measures (calculating onomasiological homogeneity and convergence/divergence between language varieties). These measures are based on onomasiological profiles, i.e. sets of alternative synonymous terms, together with their frequencies. As a development of our previous research on lexical convergence and divergence between European and Brazilian Portuguese (Soares da Silva 2010), the data include thousands of observations of the usage of alternative terms to refer to 43 football and clothing concepts. Corpus material was extracted from sports newspapers and fashion magazines from the 1950s, 1970s and 1990s/2000s, Internet chats related to football, and labels and price tags pictured from clothes shop windows. Football and clothing concepts confirm the hypothesis that the influence of foreign languages is stronger in the Brazilian variety than in the European variety. The use of loanwords has contributed towards onomasiological heterogeneity within and across the two national varieties in the last 60 years. 
[1] NOVAS PERSPETIVAS DE ESTUDO DO EMPRÉSTIMO LEXICAL

O estudo do empréstimo lexical em geral tem uma longa tradição em linguística particularmente histórica. Atualmente, são os anglicismos que despertam maior interesse, naturalmente pela enorme influência do inglês, e os estudos têm-se concentrado nos contextos de contacto linguístico ténue, como os que ocorrem na Europa ocidental, em que o contacto com o inglês se faz sobretudo através dos media.

Apesar de o empréstimo lexical ser, ao longo do século passado, um tópico relevante de investigação em linguística histórica e linguística de contacto de línguas, as questões de investigação da maior parte dos estudos existentes são de âmbito limitado. Predominantemente orientados por uma perspetiva estruturalista, os estudos têm-se concentrado na classificação de tipos de estrangeirismos de acordo com o grau da sua adaptação morfológica e fonológica à língua recetora, na sua evolução diacrónica e no seu tratamento lexicográfico. A investigação estruturalista sobre o empréstimo lexical tem estado, assim, centrada na palavra e suas formas e nos processos estruturais a que os estrangeirismos estão sujeitos.

Recentemente, novas perspetivas e agendas têm sido introduzidas, graças a estudos tipológicos (e.g. Haspelmath 2008; Haspelmath \& Tadmor 2009 e, particularmente, estudos orientados no enquadramento da (Socio)Linguística Cognitiva (ver, sobretudo, Zenner et al. 2012; Zenner 2013; Zenner \& Kristiansen 2014). Destacam-se três importantes contributos da perspetiva sociocognitiva para o estudo do empréstimo lexical, que serão desenvolvidos nas duas secções seguintes. Primeiro, os estrangeirismos são estudados a partir dos conceitos que designam, o que conduz a uma abordagem onomasiológica do empréstimo lexical: passa-se, assim, de uma perspetiva centrada na palavra para uma perspetiva centrada no conceito. Segundo, o fenómeno do empréstimo lexical incide também em unidades multipalavras, expressões idiomáticas e outras expressões do domínio da fraseologia: passa-se, pois, de uma perspetiva centrada na unidade lexical isolada para uma perspetiva construcional e contextual. Terceiro, os estrangeirismos são estudados em vastos corpora e através de avançados métodos quantitativos multivariacionais, que permitem analisar a interação entre fatores conceptuais, estruturais, socioculturais e pragmático-discursivos do empréstimo lexical e medir o sucesso dos estrangeirismos e o seu impacto na variação intralinguística: passase, assim, de uma perspetiva sistémica para uma perspetiva centrada no uso efetivo dos estrangeirismos.

O objetivo do presente estudo é apresentar o novo modelo sociocognitivo e socioletomético centrado no conceito de investigação do empréstimo lexical e aplicálo ao português como língua pluricêntrica, comparando o sucesso dos estrangeirismos nas duas variedades nacionais do português europeu (PE) e português brasileiro (PB). Nas duas secções seguintes, apresentaremos os elementos principais e os contributos da perspetiva sociocognitiva e da abordagem onomasiológica e 
socioletométrica do empréstimo lexical. Seguidamente, serão apresentados os resultados do estudo de caso sobre o uso e o sucesso de estrangeirismos que designam conceitos de futebol e de vestuário nas duas variedades nacionais do português ao longo dos últimos 60 anos e em diferentes variedades estilísticas. Com base num vasto corpus de textos de jornais desportivos e revistas de moda e aplicando métodos socioletométricos baseados em perfis onomasiológicos, procuraremos (i) medir e comparar o sucesso dos estrangeirismos no PE e no PB; (ii) confirmar a hipótese de que a influência do inglês e de outras línguas estrangeiras é maior no PB do que no PE; e (iii) determinar o impacto dos estrangeirismos na variação onomasiológica dos conceitos de futebol e vestuário e na heterogeneidade onomasiológica em geral e ainda, embora sumariamente, na relação diacrónica entre PE e PB. Este estudo é uma extensão da nossa investigação sociolexicológica, sociocognitiva e socioletométrica sobre convergência e divergência entre PE e PB (Soares da Silva 2010, 2011, 2012, 2013, 2014a,c). O estudo do impacto dos estrangeirismos na convergência e divergência diacrónica entre as duas variedades nacionais ao longo dos últimos 60 anos é desenvolvido em Soares da Silva (2014a), com base numa análise de corpus e numa análise de atitudes linguísticas.

[2] SOCIOLINGUístic A COGNitiva, CONTACTO DE LínguAs E EMPRÉstimo

A Sociolinguística Cognitiva (Kristiansen \& Dirven 2008; Croft 2009; Soares da Silva 2009; Geeraerts 2010; Geeraerts et al. 2010; Soares da Silva 2014b) é uma extensão emergente da Linguística Cognitiva (Geeraerts \& Cuyckens 2007) como modelo orientado para o significado e centrado no uso, que pretende investigar a inter-relação entre as dimensões sociais e as dimensões conceptuais da variação intralinguística através de avançados métodos empíricos quantitativos e multivariacionais. Representa a convergência de interesses de investigação da Sociolinguística e da Linguística Cognitiva e contribui quer para integrar na agenda da Linguística Cognitiva os aspetos sociais da linguagem quer para incorporar na agenda da Sociolinguística os aspetos conceptuais da variação intralinguística. A contribuição da Sociolinguística Cognitiva para o estudo das línguas pluricêntricas está patente em Soares da Silva (2014b).

Destacam-se três contributos específicos da Sociolinguística Cognitiva para a investigação sociolinguística, que evidenciam a importância da semântica nos estudos variacionistas: (i) a análise da variação do significado, isto é, os vários modos de interação entre o significado e as outras fontes de variação linguística, nomeadamente forma e contexto; (ii) o tratamento do problema metodológico da equivalência semântica, pré-requisito para o estudo sócio-variacionista do léxico e da gramática; e (iii) o estudo do significado da variação ou representação cognitiva da variação intralinguística, nas suas componentes de perceção, categorização e avaliação atitudinal da diversidade linguística. 
Em relação a (i), a distinção elaborada em semântica lexical cognitiva entre variação semasiológica (diferentes referentes/sentidos de uma palavra), variação onomasiológica conceptual (diferentes palavras conceptualmente diferentes designativas de um mesmo conceito), variação onomasiológica formal (diferentes palavras conceptualmente equivalentes designativas de um mesmo conceito) e variação contextual (diferenças sociolinguísticas, estilísticas e pragmáticas), a que voltaremos na secção seguinte, contribui para determinar a correlação entre significado, forma e contexto como fatores de variação linguística.

Quanto a (ii), a resposta à questão da equivalência semântica e, logo, à noção de variável sociolinguística passa pela incorporação de uma teoria do significado entendido como conceptualização, tal como tem sido desenvolvida pela Linguística Cognitiva, e pela verificação de que se as diferenças semânticas entre as variantes lexicais ou gramaticais forem estáveis entre variedades linguísticas, então qualquer variação remanescente é variação sociolinguística.

Relativamente a (iii), áreas da Linguística Cognitiva como a teoria do protótipo e a teoria dos modelos cognitivos e culturais permitem verificar como os falantes percecionam, categorizam e avaliam as variedades linguísticas.

Mais diretamente em relação à linguística de contacto de línguas (Matras 2009) e a fenómenos induzidos por esse contacto como os empréstimos, destacamos quatro contributos associados à aplicação de modelos da Linguística Cognitiva.

A Teoria do Protótipo (Geeraerts et al. 1994; Taylor 1995) mostra que empréstimo e mudança de código («codeswitching») não se deixam definir em termos de propriedades necessárias e suficientes, mas com base em protótipos e efeitos de prototipicidade, pelo que os empréstimos evidenciam diferentes graus de saliência cognitiva e os limites entre empréstimo e mudança de código são difusos.

As noções de saliência cognitiva e de incrustamento («entrenchment») (Schmid 2007) permitem compreender a variação no fenómeno do empréstimo e o papel do significado conceptual, pragmático e social na promoção da atratividade de determinadas palavras de uma língua estrangeira.

A Gramática de Construções (Langacker 1987; Goldberg 1995; Croft 2001) contribui para integrar as tradições de investigação sobre empréstimo lexical, empréstimo gramatical e mudança de código, introduzindo a ideia de um contínuo entre léxico e gramática.

A Teoria da Metáfora Conceptual e dos Modelos Cognitivos Culturais (Lakoff \& Johnson 1980, 1999) é útil para o estudo das diferenças (inter)culturais dentro e entre línguas em contacto, bem como para o estudo da perceção, categorização e avaliação atitudinal do contacto de línguas e dos empréstimos.

Os contributos da Sociolinguística Cognitiva para o estudo do empréstimo lexical estão patentes em Zenner \& Kristiansen (2014). 
[3] MÉTODO ONOMASIOLÓGICO E SOCIOLETOMÉTRICO

Utilizamos neste estudo uma perspetiva onomasiológica de investigação do empréstimo lexical, no sentido de que tomamos o conceito expresso pelo estrangeirismo como ponto de partida. A análise incide na variação onomasiológica entre palavras semanticamente equivalentes (sinónimos denotacionais), de que o estrangeirismo faz parte. Lembremos a distinção clássica entre semasiologia e onomasiologia, estabelecida na tradição europeia da semântica lexical (Baldinger 1964). Enquanto a perspetiva semasiológica toma a palavra como ponto de partida para analisar os seus vários sentidos ou referentes, a perspetiva onomasiológica parte do conceito para analisar as diferentes palavras ou outras expressões que o designam. A semasiologia ocupa-se de fenómenos como a polissemia (Soares da Silva 2006), ao passo que a onomasiologia estuda fenómenos como a sinonímia e mecanismos lexicogenéticos como a formação de palavras ou o empréstimo.

A variação onomasiológica pode envolver diferenças conceptuais e/ou diferenças sociais. Assim, as escolhas lexicais podem ser determinadas ora por fatores conceptuais ora por fatores dialetais, socioletais ou idioletais - numa palavra, letais. Por exemplo, a opção entre guarda-redes e goleiro ou equipa e time é uma escolha de formas que exprimem o mesmo conceito mas pertencem a diferentes variedades nacionais; e a opção entre morrer e falecer é uma escolha de formas que exprimem o mesmo conceito mas são estilisticamente diferenciadas. Podemos designar esta variação entre sinónimos denotacionais como variação onomasiológica formal, em oposição à variação onomasiológica conceptual, como a que se dá, por exemplo, entre guarda-redes e jogador (sendo o primeiro termo hipónimo do segundo), a qual envolve diferenças conceptuais (Geeraerts et al. 1994). A variação onomasiológica formal deve-se, pois, não a uma classificação conceptual diferente da mesma entidade, mas ao uso de diferentes palavras referindo o mesmo conceito e associadas a diferentes regiões, grupos sociais ou registos, isto é, os sinónimos denotacionais. Esta variação onomasiológica é sociolinguisticamente relevante, justamente porque os sinónimos denotacionais revelam a própria existência e competição entre variedades letais.

Convém notar que a distinção entre variação onomasiológica formal e conceptual não é dicotómica, bem como não é fácil estabelecer uma relação de equivalência semântica entre diferentes expressões. Na verdade, podem existir diferenças conceptuais subtis. Em relação a itens lexicais concretos, a equivalência semântica é mais fácil de estabelecer, na medida em que podemos controlar os referentes e assim verificar se o referente é o mesmo ou não. Neste estudo, os sinónimos denotacionais de peças de vestuário foram determinados com base em fotos das respetivas peças; no caso dos termos de futebol, as imagens e/ou o contexto permitiram determinar objetivamente a relação de sinonímia denotacional. As dificuldades aumentam quando passamos de itens lexicais concretos para itens abstratos e para construções gramaticais. Todavia, o que importa determinar não 
é se as expressões alternativas diferem semanticamente, mas se as diferenças semânticas são estáveis entre as diferentes variedades.

O conjunto das expressões sinónimas alternativas usadas para designar determinado conceito, juntamente com as suas frequências relativas no corpus, constitui o perfil onomasiológico desse conceito. Por exemplo, o perfil onomasiológico de GOLO inclui os termos alternativos bola, goal, gol, gôl, golo, ponto e tento. A Tabela 1 apresenta as frequências absolutas e relativas de cada um dos termos alternativos nas bases de dados do PE e do PB na década de 50 .

O método onomasiológico tem vantagens no estudo da variação intralinguística, bem como no estudo do empréstimo lexical. Analisar expressões sinónimas alternativas de conceitos lexicais permite um mecanismo de controlo capaz de evitar quer os perigos estatísticos resultantes de uma distribuição assimétrica de conceitos quer os perigos temáticos no corpus. Na verdade, as frequências dos termos no corpus podem estar correlacionadas tanto com a preferência onomasiológica formal como com a especificidade temática. Tomando o perfil onomasiológico de CASACO, o eventual predomínio do termo casaco pode indicar uma preferência pelo termo casaco em detrimento de blazer, mas pode também resultar do facto de os textos evitarem o uso de estrangeirismos (ou então indicar que os termos não são sinónimos). Um outro exemplo: comparando o uso de estrangeirismos em diferentes períodos temporais, o aumento da sua frequência não significa necessariamente maior sucesso desses estrangeirismos, pois pode ter resultado do facto de o corpus mais recente ter mais textos de um assunto propício a estrangeirismos do que o corpus mais antigo.

A perspetiva onomasiológica de estudo do empréstimo lexical tem a vantagem de se deixar de olhar apenas para o termo estrangeiro e de se passar a considerar o termo estrangeiro na sua relação com o conceito que designa e com os outros termos que exprimem esse conceito. Outra vantagem é a redefinição da distinção entre empréstimo estrito e empréstimo cultural ou, o mesmo é dizer, empréstimo supérfluo e empréstimo necessário: os empréstimos culturais ou necessários designam objetos ou conceitos novos (CD ou compact disk, blog, start-up), sendo portanto a única lexicalização de determinado conceito, ao passo que os empréstimos estritos ou supérfluos duplicam termos já existentes, passando assim a competir com esses termos vernáculos (ranking, manager). A abordagem onomasiológica permite ainda medir o sucesso dos estrangeirismos, expresso na sua preferência para designar os respetivos conceitos relativamente aos termos sinónimos com que competem.

Para medir o sucesso dos estrangeirismos e o seu impacto na variação intralinguística, são usados três métodos socioletométricos baseados em perfis onomasiológicos: medida da proporção de determinada característica (A), medida de uniformidade interna (I) e medida de uniformidade externa (U). Estas medidas foram desenvolvidas pela unidade de investigação Quantitative Lexicology and Vari- 


\begin{tabular}{|c|c|c|c|c|c|c|c|}
\hline \multirow{2}{*}{ GOLO } & \multicolumn{3}{|c|}{ P50 } & \multicolumn{3}{|c|}{ B50 } & \multirow[b]{2}{*}{ W } \\
\hline & abs & rel & rel*W $^{*}$ & abs & rel & rel*W & \\
\hline bola & 109 & 3,7 & 0,0 & 0 & 0,0 & 0,0 & 0 \\
\hline goal & 24 & 0,8 & 0,8 & 528 & 38,8 & 38,8 & 1 \\
\hline gol & 0 & 0,0 & 0,0 & 111 & 8,1 & 4,1 & 0,5 \\
\hline gôl & 0 & 0,0 & 0,0 & 66 & 4,8 & 1,9 & 0,4 \\
\hline golo & 1841 & 61,9 & 31,0 & 0 & 0,0 & 0,0 & 0,5 \\
\hline ponto & 204 & 6,9 & 0,0 & 26 & 1,9 & 0,0 & 0 \\
\hline tento & 795 & 26,7 & 0,0 & 631 & 46,3 & 0,0 & 0 \\
\hline $\mathbf{A}_{I n g}$ & & & 31,8 & & & 44,8 & \\
\hline
\end{tabular}

TABELA 1: Impacto de anglicismos $\left(\mathrm{A}_{\text {Ing }}\right)$ no perfil GOLO no PE e PB nos anos 50

ational Linguistics (QLVL) no seu estudo socioletométrico do neerlandês da Bélgica e da Holanda (Geeraerts et al. 1999; Speelman et al. 2003). Para cada um destes cálculos, são efetuadas medidas não ponderadas (A, I, U) e ponderadas ( $\left.A^{\prime}, I^{\prime}, U^{\prime}\right)$. As primeiras têm em conta a frequência relativa de cada conceito no corpus. Neste estudo, o impacto dos estrangeirismos é considerado do ponto de vista pragmático e comunicativo, atendendo a diferenças de frequência dos conceitos estudados, e não do ponto de vista estrutural, que atribui o mesmo peso a cada conceito. Por isso, as medidas ponderadas são estatisticamente mais significativas.

A medida A/A' é a medida fundamental neste estudo: permite calcular a proporção de termos com determinada característica - neste caso, a proporção de estrangeirismos - no perfil onomasiológico de um conceito e nos perfis onomasiológicos de um conjunto de conceitos na amostra estudada. A característica $\mathrm{K}$ - estrangeirismo, neste caso - é um contínuo. Assim, é atribuído o valor mais alto (1) aos estrangeirismos que mantêm a forma original e o valor mais baixo $(0,25)$ a fortes adaptações e a decalques. A proporção (A) de todos os estrangeirismos usados para nomear um conceito é a soma das frequências relativas dos estrangeirismos atestados no corpus ponderada pelo valor $(W)$ de adaptação dos estrangeirismos. Tomando o exemplo do perfil GOLO, é atribuído o valor (1) ao termo goal, visto que mantém a forma gráfica e fonética do termo original do inglês, e o valor $(0,5)$ a golo (usado no PE) e gol (usado no PB). Na Tabela 1, é calculada a influência do inglês no perfil onomasiológico de GOLO no PE e no PB dos anos 50 (P50, B50), com base nas frequências absolutas (abs) e relativas (rel) dos termos alternativos, no valor $(\mathrm{W})$ de anglicismo e na soma das frequências relativas dos termos alternativos ponderada pelo valor $\mathrm{W}\left(\right.$ rel $\left.^{*} \mathrm{~W}\right)$. A proporção de anglicismos no perfil de GOLO na década de 50 é maior no PB $(44,8 \%)$ do que no PE $(31,8 \%)$. 


\begin{tabular}{lcccc}
\hline AVANÇADO & P50 & $\mathbf{( P 5 0 )}^{2}$ & B50 & (B50) $^{2}$ \\
\hline atacante & 8,8 & 77,8 & 36,6 & 1340,7 \\
avançado & 71,6 & 5128,8 & 0,9 & 0,9 \\
avante & 0,0 & 0,0 & 48,9 & 2393,5 \\
dianteiro & 19,2 & 369,2 & 6,8 & 45,8 \\
forward & 0,1 & 0,0 & 5,2 & 27,4 \\
ponta de lança & 0,3 & 0,1 & 1,5 & 2,4 \\
\hline I & & $\mathbf{5 5 , 8}$ & & $\mathbf{3 8 , 1}$ \\
\hline
\end{tabular}

TABELA 2: Uniformidade interna (I) do perfil AVANÇADO em PE e PB nos anos 50

A medida de uniformidade interna (I/I') consiste em calcular a uniformidade dentro de uma única variedade linguística: I/I' atinge o seu valor mais elevado quando todos os falantes escolhem o mesmo item lexical para designar determinado conceito; diminui à medida que aumentar ou o número de termos alternativos ou o número de termos dominantes. A uniformidade interna de um conceito é igual à soma dos quadrados das frequências relativas dos itens lexicais usados para nomear esse conceito. Tomando o exemplo do perfil de AVANÇADO, a Tabela 2 mostra que a uniformidade interna na década de 50 é maior no PE $(55,8 \%)$ do que no PB $(38,1 \%)$. I/I' é um indicador de homo/heterogeneidade onomasiológica dentro e entre variedades linguísticas.

A medida de uniformidade externa (U/U') consiste em calcular a uniformidade entre diferentes variedades linguísticas. A uniformidade externa de um conceito entre duas variedades é igual à soma das frequências relativas mais pequenas dos itens lexicais usados para designar esse conceito. Diacronicamente, convergência e divergência entre duas variedades traduzem-se em aumento e diminuição da uniformidade externa, respetivamente. Sincronicamente, quanto maior é a distância entre os registos padrão e subpadrão, menor é a uniformidade externa entre esses dois registos. Dada a pouca relevância desta medida no presente estudo, não a vamos exemplificar (ver Soares da Silva 2010).

\section{[4] CORPUS E BASE DE DADOS}

Os dados linguísticos para o presente estudo foram recolhidos dos campos lexicais do futebol e da moda/vestuário, devido à popularidade dos respetivos conceitos e ao facto de serem suscetíveis à influência de línguas estrangeiras. Os materiais do corpus foram extraídos de três fontes: (i) jornais de desporto e revistas de moda dos primeiros anos das décadas de 50, 70 e 90-2000; (ii) linguagem da Internet de chats associados a clubes de futebol; e (iii) etiquetas de roupas de lojas de vestuário. Os materiais de (i) permitem responder à questão diacrónica de saber se a influência das línguas estrangeiras é maior no PB ou no PE e se aumentou ou 
diminuiu ao longo do tempo, bem como à questão da convergência e divergência entre PE e PB. Os materiais de (ii) e (iii), de diferentes fontes pela maior produtividade de cada uma, mas ambos de um registo mais informal, permitem saber em que estrato é maior a influência das línguas estrangeiras, bem como estudar a estratificação de cada uma das variedades nacionais. Os dados relativos ao PB provêm de São Paulo e do Rio de Janeiro.

O subcorpus de futebol contém 2,7 milhões de palavras oriundas de 8 jornais desportivos (4 portugueses e 4 brasileiros) e 15 milhões de palavras coligidas de chats da Internet. 0 subcorpus de vestuário contém 1,2 milhões de palavras de 28 revistas de moda (14 portuguesas e 14 brasileiras) e 1300 imagens de etiquetas de lojas de vestuário. Estes dois subcorpora constituem o corpus CONDIVport (Soares da Silva 2008), disponibilizado na Linguateca, em www . linguateca. pt/ACDC (Santos \& Sarmento 2003).

Com base no corpus CONDIVport, coligimos os sinónimos denotacionais de 43 conceitos nominais, sendo 21 do vocabulário do futebol e 22 do vocabulário do vestuário, juntamente com as suas frequências de uso no corpus. Para a seleção dos 43 conceitos de futebol e de vestuário foram tomados como critérios a sua heterogeneidade onomasiológica formal e a sua representatividade nos respetivos campos lexicais. Foram excluídos termos de gíria ou metafóricos, para não inflacionar as diferenças entre as duas variedades nacionais. Os 21 conceitos ou perfis onomasiológicos de futebol, perfazendo um total de 183 termos, foram analisados numa base de dados constituída por 90.202 observações do uso destes termos nos jornais desportivos portugueses e brasileiros selecionados e 143.946 observações do seu uso nos chats. Os 22 perfis onomasiológicos de vestuário de homem (M) e de mulher (F), num total de 264 termos, foram analisados numa base de dados de 12.451 observações do seu uso nas revistas de moda portuguesas e brasileiras selecionadas e em 2.775 observações do seu uso em etiquetas de roupas de lojas de vestuário. Todos os perfis de futebol e vestuário, incluindo os sinónimos denotacionais selecionados e observados no corpus, são apresentados em Anexo. Os estrangeirismos que mantêm a sua forma original estão indicados entre aspas. 0 número total de observações no corpus CONDIVport de cada perfil de futebol e de vestuário encontra-se em Soares da Silva $(2010,2014 a)$.

\section{[5] RESULTADOS}

\section{[5.1] Sucesso e evolução dos estrangeirismos no PE e no PB}

Tomando a medida A/A', descrita na secção [3], vamos quantificar a proporção de termos com a característica 'anglicismo', 'francesismo' ou 'estrangeirismo' (qualquer que seja a proveniência) dentro do perfil onomasiológico de determinado conceito e, depois, no conjunto dos conceitos analisados nas amostras das duas variedades. Os estrangeirismos no vocabulário de futebol são distribuídos por dois conjuntos: anglicismos e todos os estrangeirismos (incluindo espanholismos, ita- 


\begin{tabular}{|c|c|c|c|c|}
\hline $\mathrm{A}_{\text {Ing }}(\mathrm{P} 50)$ & $7,1 \%$ & $<$ & $\mathrm{A}^{\prime}{ }_{I n g}(\mathrm{~B} 50)$ & $18 \%$ \\
\hline $\mathrm{A}_{\text {Ing }}^{\prime}$ (P70) & $9,8 \%$ & $<$ & $\mathrm{A}_{\text {Ing }}^{\prime}(\mathrm{B} 70)$ & $17,1 \%$ \\
\hline $\mathrm{A}_{\text {Ing }}^{\prime}(\mathrm{P} 00)$ & $10,2 \%$ & $<$ & $\mathrm{A}_{I n g}^{\prime}(\mathrm{B} 00)$ & $16,2 \%$ \\
\hline$\overline{\mathrm{A}}_{\text {Ing }} \overline{(\mathrm{P} 50)}$ & $13,2 \%$ & $<$ & $\overline{\mathrm{A}}_{\text {Ing }}(\overline{\mathrm{B}} \overline{50}) \overline{\mathrm{s}}$ & $1 \overline{8}, \overline{5} \%$ \\
\hline $\mathrm{A}_{\text {Ing }}(\mathrm{P} 70)$ & $15 \%$ & $<$ & $\mathrm{A}_{\text {Ing }}(\mathrm{B} 70)$ & $20,4 \%$ \\
\hline $\mathrm{A}_{\text {Ing }}(\mathrm{P} 00)$ & $12,8 \%$ & $<$ & $\mathrm{A}_{I n g}(\mathrm{~B} 00)$ & $20,3 \%$ \\
\hline $\mathrm{A}_{\text {estr }}$ (P50) & $13,9 \%$ & $<$ & $\mathrm{A}_{\text {estr }}$ (B50) & $23,5 \%$ \\
\hline $\mathrm{A}^{\prime}{ }_{\text {estr }}$ (P70) & $17,9 \%$ & $<$ & $\mathrm{A}_{\text {estr }}^{\prime}$ (B70) & $22,8 \%$ \\
\hline $\mathrm{A}^{\prime}$ estr $(\mathrm{P} 00)$ & $18,5 \%$ & $<$ & $\mathrm{A}^{\prime}{ }_{\text {estr }}(\mathrm{B} 00)$ & $23,3 \%$ \\
\hline $\mathrm{A}_{\text {estr }}(\mathrm{P} 50)$ & $15,9 \%$ & $<$ & $\overline{\mathrm{A}}_{e s t r}(\overline{\mathrm{B}} 50)$ & $23,4 \%$ \\
\hline $\mathrm{A}_{\text {estr }}$ (P70) & $18 \%$ & $<$ & $\mathrm{A}_{e s t r}(\mathrm{~B} 70)$ & $25 \%$ \\
\hline $\mathrm{A}_{\text {estr }}(\mathrm{P} 00)$ & $15,9 \%$ & $<$ & $\mathrm{A}_{e s t r}(\mathrm{~B} 00)$ & $25 \%$ \\
\hline
\end{tabular}

TABELA 3: Estrangeirismos no corpus de futebol (de 1950 a 2000)

lianismos e galicismos). Os estrangeirismos no vocabulário do vestuário são distribuídos por três conjuntos: galicismos, anglicismos e todos os estrangeirismos.

As Tabelas $3 \mathrm{e} 4$ apresentam os resultados com as percentagens de anglicismos $\left(\mathrm{A} / \mathrm{A}^{\prime}{ }_{\text {Ing }}\right)$, francesismos $\left(\mathrm{A} / \mathrm{A}_{F r}\right)$ e todos os estrangeirismos $\left(\mathrm{A} / \mathrm{A}^{\prime}{ }_{\text {estr }}\right)$ no $\mathrm{PE}(\mathrm{P}) \mathrm{e}$ PB (B), nos três períodos em análise (1950, 1970 e 2000). É apresentada, primeiramente, a proporção ponderada (A') e, depois, a proporção não ponderada (A) de estrangeirismos.

No futebol, a influência de anglicismos e outros estrangeirismos é claramente maior no PB do que no PE em todos os períodos estudados. É nos anos 50 que se verifica a distância maior entre as duas variedades: mais do dobro de anglicismos em B50. Esta grande diferença percentual entre B50 e P50 resulta do facto de haver maior número e frequência de estrangeirismos conservando a sua forma original no PB. É o caso de referee, forward, back, team, foul, goal, keeper, match, half, shoot, corner, ausentes nos jornais portugueses. No PE há aumento, principalmente dos anos 50 para 70, ao passo que no PB parece haver uma pequena diminuição. Este ligeiro decréscimo resulta, porém, dos aportuguesamentos dos estrangeirismos no PB a partir dos anos 70.

No vestuário, verifica-se uma diminuição de francesismos e um claro aumento de anglicismos, por razões socioculturais bem conhecidas, designadamente a diminuição da influência do francês e o inevitável aumento da influência do inglês. No conjunto de todos os estrangeirismos no vocabulário do vestuário, temos um aumento no PE e uma situação de estabilidade no PB. A influência de estrangeirismos continua a ser maior no $\mathrm{PB}$, embora a diferença entre as duas variedades seja menos acentuada. 


\begin{tabular}{|c|c|c|c|c|}
\hline $\mathrm{A}_{F r}^{\prime}(\mathrm{P} 50)$ & $17,6 \%$ & $\cong$ & $\mathrm{A}_{F r}^{\prime}(\mathrm{B} 50)$ & $18,5 \%$ \\
\hline $\mathrm{A}_{F r}^{\prime}(\mathrm{P} 70)$ & $15,9 \%$ & $\cong$ & $\mathrm{A}_{F r}^{\prime}(\mathrm{B} 70)$ & $18,1 \%$ \\
\hline $\mathrm{A}^{\prime}{ }_{F r}(\mathrm{P} 00)$ & $10,2 \%$ & $\cong$ & $\mathrm{A}^{\prime}{ }_{F r}(\mathrm{~B} 00)$ & $7,9 \%$ \\
\hline$\left.\overline{\mathrm{A}}_{F r}^{-}-\overline{\mathrm{P}} \overline{50}\right) \overline{-}$ & $16, \overline{7} \%$ & $<$ & $\overline{\mathrm{A}}_{F r}(\overline{\mathrm{B}} 5 \overline{0})$ & $2 \overline{2}, \overline{2} \%$ \\
\hline $\mathrm{A}_{F r}(\mathrm{P} 70)$ & $20,6 \%$ & $\cong$ & $\mathrm{A}_{F r}(\mathrm{~B} 70)$ & $17,6 \%$ \\
\hline $\mathrm{A}_{F r}(\mathrm{P} 00)$ & $16,1 \%$ & $\cong$ & $\mathrm{A}_{F r}(\mathrm{~B} 00)$ & $16,9 \%$ \\
\hline $\mathrm{A}_{\text {Ing }}^{\prime}$ (P50) & $3,3 \%$ & $\cong$ & $\mathrm{A}_{\text {Ing }}^{\prime}$ (B50) & $4,2 \%$ \\
\hline $\mathrm{A}_{\text {Ing }}^{\prime}(\mathrm{P} 70)$ & $5,8 \%$ & $\cong$ & $\mathrm{A}^{\prime}{ }_{I n g}(\mathrm{~B} 70)$ & $7,6 \%$ \\
\hline $\mathrm{A}^{\prime}{ }_{I n g}(\mathrm{P} 00)$ & $16,9 \%$ & $\cong$ & $\mathrm{A}_{\text {Ing }}^{\prime}(\mathrm{B} 00)$ & $16,8 \%$ \\
\hline$\overline{\mathrm{A}}_{I n g}(\overline{\mathrm{P}} \overline{50})$ & $7,7 \%$ & $\cong$ & $\overline{\mathrm{A}}_{I n g}(\overline{\mathrm{B}} \overline{50})$ & $6,7 \%$ \\
\hline $\mathrm{A}_{\text {Ing }}(\mathrm{P} 70)$ & $16 \%$ & $\cong$ & $\mathrm{A}_{\text {Ing }}(\mathrm{B} 70)$ & $15 \%$ \\
\hline $\mathrm{A}_{\text {Ing }}(\mathrm{P} 00)$ & $19,5 \%$ & $<$ & $\mathrm{A}_{\text {Ing }}(\mathrm{B} 00)$ & $27,1 \%$ \\
\hline $\mathrm{A}_{\text {estr }}^{\prime}$ (P50) & $22,4 \%$ & $\cong$ & $\mathrm{A}_{\text {estr }}$ (B50) & $23,8 \%$ \\
\hline $\mathrm{A}_{\text {estr }}^{\prime}$ (P70) & $22,1 \%$ & $\cong$ & $\mathrm{A}_{\text {estr }}^{\prime}$ (B70) & $26,7 \%$ \\
\hline $\mathrm{A}_{\text {estr }}^{\prime}(\mathrm{P} 00)$ & $28,2 \%$ & $\cong$ & $\mathrm{A}_{\text {estr }}^{\prime}(\mathrm{B} 00)$ & $24,9 \%$ \\
\hline$\overline{\mathrm{A}}_{\text {estr }}^{-} \overline{(\mathrm{P} 50)}$ & $27,3 \%$ & $<$ & $\overline{\mathrm{A}}_{\text {estr }}^{-}(\overline{\mathrm{B}} \overline{50})$ & $3 \overline{3} \%$ \\
\hline $\mathrm{A}_{\text {estr }}$ (P70) & $38,1 \%$ & $\cong$ & $\mathrm{A}_{\text {estr }}$ (B70) & $34,4 \%$ \\
\hline $\mathrm{A}_{e s t r}(\mathrm{P} 00)$ & $37,9 \%$ & $<$ & $\mathrm{A}_{e s t r}(\mathrm{~B} 00)$ & $44,4 \%$ \\
\hline
\end{tabular}

TABELA 4: Estrangeirismos no corpus de vestuário (de 1950 a 2000)

No conjunto dos dois campos lexicais, a influência do inglês aumenta e é maior no PB (embora haja uma pequena descida desta influência no PB no campo do futebol). Por seu lado, a influência do francês diminui (embora esta influência seja muito pouco significativa no domínio do futebol) $\mathrm{e}-$ facto interessante a registar - essa influência não é menos importante no PB do que no PE. As Tabelas 3 e 4 confirmam, assim, a hipótese de uma maior permeabilidade do $\mathrm{PB}$ aos estrangeirismos, quer importando-os diretamente, quer adaptando-os.

Até aqui temos os resultados globais do impacto dos estrangeirismos em cada uma das variedades nacionais. Importa também olhar para o comportamento dos perfis individuais e comparar o seu impacto nos resultados globais. A questão é saber se a proporção de estrangeirismos dos perfis individuais segue ou não a tendência global. Por limitações de espaço, não vamos analisar aqui esta questão. Em Soares da Silva (2014a), pode verificar-se que o desvio padrão é geralmente maior no PB do que no PE. Por outro lado, entre os perfis com maior proporção de estrangeirismos encontram-se tanto conceitos mais frequentes como menos frequentes.

Vejamos agora a questão da adaptação de estrangeirismos. A Tabela 5 evidencia a maior tendência do $\mathrm{PB}$ (B) à adaptação, em contraste com o PE (P), que tende para a substituição por termos vernáculos. A percentagem de adaptações 


\begin{tabular}{|c|c|c|c|c|}
\hline $\mathrm{A}_{\text {Ing.adapt }}$ (P50) & $6 \%$ & $\cong$ & $\mathrm{A}_{\text {Ing.adapt }}^{\prime}$ (B50) & $2,8 \%$ \\
\hline $\mathrm{A}_{\text {Ing.adapt }}^{\prime}$ (P70) & $7,9 \%$ & $<$ & $\mathrm{A}_{\text {Ing.adapt }}^{\prime}(\mathrm{B} 70)$ & $16,9 \%$ \\
\hline $\mathrm{A}^{\prime}$ Ing.adapt $(\mathrm{PO0})$ & $8,9 \%$ & $<$ & $\mathrm{A}^{\prime}$ Ing.adapt $(\mathrm{B} 00)$ & $16 \%$ \\
\hline$\overline{\mathrm{A}}_{\text {Ing.adapt }}(\overline{\mathrm{P}} 50)$ & $7,5 \%$ & $\cong$ & $\overline{\mathrm{A}}_{\text {Ing.adapt }}(\overline{\mathrm{B}} 50)$ & $3,8 \%$ \\
\hline $\mathrm{A}_{\text {Ing.adapt }}(\mathrm{P} 70)$ & $7,5 \%$ & $<$ & $\mathrm{A}_{\text {Ing.adapt }}$ (B70) & $16,5 \%$ \\
\hline $\mathrm{A}_{\text {Ing.adapt }}(\mathrm{P} 00)$ & $7,8 \%$ & $<$ & $\mathrm{A}_{\text {Ing.adapt }}$ (B00) & $15,8 \%$ \\
\hline
\end{tabular}

TABELA 5: Adaptações/decalques de anglicismos no corpus de futebol

de termos ingleses de futebol $\left(\mathrm{A}_{\text {Ing.adapt }} / \mathrm{A}^{\prime}\right.$ Ing.adapt $)$ aumenta fortemente no $\mathrm{PB}$ de 50 para 70 . No conjunto dos 21 perfis, temos 23 adaptações e 19 decalques no PB contra 6 adaptações e 14 decalques no PE. Alguns exemplos relativamente aos anglicismos goal-keeper, goal, corner, penalty, back, shoot, offside: no PB, goleiro, gol, córner, pênalti, beque, chute e impedimento; no PE, guarda-redes, baliza, pontapé de canto, grande penalidade, defesa, pontapé/chuto e fora de jogo. A exceção é offside: decalcado em fora de jogo no PE e substituído pelo termo vernáculo impedimento no PB.

Em síntese, as Tabelas 3, 4 e 5 confirmam a hipótese de uma maior permeabilidade do $\mathrm{PB}$ aos empréstimos lexicais, quer importando-os diretamente, quer adaptando-os.

Vejamos agora o impacto dos estrangeirismos em diferentes variedades estilísticas, para saber se a influência estrangeira é maior no registo padrão ou no registo subpadrão. Para isso vamos comparar, no caso do futebol, os dados do registo mais formal dos jornais dos anos 90/2000 (P00 e B00) e os dados do registo informal dos chats $\left(\mathrm{P}_{s u b} 00\right.$ e $\left.\mathrm{B}_{s u b} 00\right)$ e, no caso do vestuário, os dados do registo mais formal e mais nacional das revistas de moda dos anos 90/2000 (P00 e B00) e os dados do registo informal e mais local das etiquetas de lojas de vestuário $\left(\mathrm{P}_{\text {sub }} 00\right.$ e $\mathrm{B}_{\text {sub }} 00$ ).

A Tabela 6 apresenta os resultados em relação aos empréstimos do inglês no vocabulário do futebol. A proporção ponderada (A') de anglicismos é maior no registo coloquial dos chats ( $14,3 \%$ no $\mathrm{PE}$ e $26,9 \%$ no $\mathrm{PB})$ do que no registo formal dos jornais (10,2\% no PE e 16,2\% no PB). A nível da medida não ponderada (A), não há diferenças significativas. A proporção $\left(A^{\prime} / A\right)$ de empréstimos do inglês no registo coloquial é também maior no PB (A' 26,9\%/A 19,7\%) do que no PE (A' 14,3\%/A 12,3\%).

As percentagens de empréstimos do inglês e do francês dos termos de vestuário estão indicadas na Tabela 7. A proporção A'/A de estrangeirismos usados nas etiquetas de lojas de vestuário é idêntica à proporção $\mathrm{A}^{\prime} / \mathrm{A}$ dos mesmos estrangeirismos usados nas revistas de moda. A única exceção ocorre no PB, mas isso deve-se principalmente às poucas ocorrências do perfil CASACO F e do termo blazer (blêizer, blêiser). A proporção de galicismos no registo subpadrão é idêntica 


\begin{tabular}{|c|c|c|c|c|}
\hline $\mathrm{A}_{\text {Ing }}(\mathrm{P} 00)$ & $10,2 \%$ & $<$ & $\mathrm{A}_{\text {Ing }}^{\prime}\left(\mathrm{P}_{\text {sub }} 00\right)$ & $14,3 \%$ \\
\hline $\mathrm{A}^{\prime}{ }_{\text {Ing }}(\mathrm{B} 00)$ & $16,2 \%$ & $<$ & $\mathrm{A}^{\prime}$ Ing $\left(\mathrm{B}_{s u b} 00\right)$ & $26,9 \%$ \\
\hline $\mathrm{A}^{\prime}{ }_{I n g}\left(\mathrm{P}_{s u b} 00\right)$ & $14,3 \%$ & $\leq$ & $\mathrm{A}^{\prime}{ }_{I n g}\left(\mathrm{~B}_{s u b} 00\right)$ & $26,9 \%$ \\
\hline$\overline{\mathrm{A}}_{\text {Ing }}(\overline{\mathrm{P} 00)}-$ & $\overline{1} \overline{8} \overline{8} \%$ & $\cong$ & $\mathrm{A}_{\text {Ing }}\left(\mathrm{P}_{\text {sub }} 00\right)$ & $1 \overline{2}, \overline{3} \%$ \\
\hline $\mathrm{A}_{\text {Ing }}(\mathrm{B} 00)$ & $20,3 \%$ & $\cong$ & $\mathrm{A}_{\text {Ing }}\left(\mathrm{B}_{s u b} 00\right)$ & $19,7 \%$ \\
\hline $\mathrm{A}_{\text {Ing }}\left(\mathrm{P}_{\text {sub }} 00\right)$ & $12,3 \%$ & $<$ & $\mathrm{A}_{\text {Ing }}\left(\mathrm{B}_{s u b} 00\right)$ & $19,7 \%$ \\
\hline
\end{tabular}

TABELA 6: Anglicismos de futebol nas variedades padrão e subpadrão

\begin{tabular}{|c|c|c|c|c|}
\hline $\mathrm{A}_{\text {Ing }}(\mathrm{P} 00)$ & $16,9 \%$ & $\cong$ & $\mathrm{A}^{\prime}$ Ing $\left(\mathrm{P}_{s u b} 00\right)$ & \\
\hline $\mathrm{A}^{\prime}{ }_{\text {Ing }}(\mathrm{B} 00)$ & $16,8 \%$ & $>$ & $\mathrm{A}^{\prime}$ Ing $\left(\mathrm{B}_{\text {sub }} 00\right)$ & 10,1 \\
\hline $\mathrm{A}^{\prime}{ }_{I n g}\left(\mathrm{P}_{s u b} 00\right)$ & $18 \%$ & $\geq$ & $\mathrm{A}^{\prime}{ }_{I n g}\left(\mathrm{~B}_{s u b} 00\right)$ & $10,1 \%$ \\
\hline$\overline{\mathrm{A}}_{\text {Ing }}(\overline{\mathrm{P}} \mathbf{0} \overline{)})$ & $19,5 \%$ & $\cong$ & $\mathrm{A}_{\text {Ing }}\left(\mathrm{P}_{s u b} 00\right)$ & $1 \overline{7}, \overline{9} \%$ \\
\hline $\mathrm{A}_{\text {Ing }}(\mathrm{B} 00)$ & 27,1 & $>$ & $\mathrm{A}_{\text {Ing }}\left(\mathrm{B}_{s l}\right.$ & $22,6 \%$ \\
\hline $\mathrm{A}_{\text {Ing }}\left(\mathrm{P}_{s u b} 0 \mathrm{C}\right.$ & $17,9 \%$ & $<$ & $\mathrm{A}_{\text {Ing }}\left(\mathrm{B}_{s}\right.$ & $22,6 \%$ \\
\hline & $10,2 \%$ & $\cong$ & $\mathrm{A}_{F r}^{\prime}\left(\mathrm{P}_{s}\right.$ & 9,7 \\
\hline $\mathrm{A}_{F r}^{\prime}(\mathrm{B} 00)$ & $7,9 \%$ & $\cong$ & $\mathrm{A}^{\prime}{ }_{F r}\left(\mathrm{~B}_{s u}\right.$ & $10,4 \%$ \\
\hline $\mathrm{A}^{\prime}{ }_{F r}\left(\mathrm{P}_{s u b} 00\right)$ & $9,7 \%$ & $\cong$ & & 10,4 \\
\hline$(\overline{\mathrm{P}} 00)^{--}$ & $\overline{16}, \overline{1 \%}$ & $\cong$ & & $14, \overline{1} \%$ \\
\hline & $16,9 \%$ & $>$ & & $11,3 \%$ \\
\hline $\mathrm{A}_{F r}\left(\mathrm{P}_{s u b} 00\right.$ & $14,1 \%$ & $\cong$ & $\mathrm{A}_{F r}\left(\mathrm{~B}_{s u b} 00\right)$ & $11,3 \%$ \\
\hline
\end{tabular}

TABELA 7: Anglicismos e francesismos de vestuário nas variedades padrão e subpadrão

nas duas variedades nacionais. Já a proporção ponderada (A') de anglicismos no registo subpadrão é maior no PE (18\%) do que no PB $(10,1 \%)$, embora tal se deva à ausência de ocorrências de $t$-shirt nas lojas brasileiras de vestuário. Mas a nível da medida não ponderada (A), a proporção de anglicismos é maior no PB $(22,6 \%)$ do que no PE $(17,9 \%)$.

A comparação estratificacional sincrónica sugere dois resultados. Primeiro, no vocabulário de futebol, o registo subpadrão parece ser mais recetivo a estrangeirismos do que o registo padrão. Trata-se de um efeito da globalização dos termos de futebol. Obviamente que a globalização tem influência em ambos os registos, mas o seu impacto no registo subpadrão é maior devido à grande popularidade do futebol e à sua enorme difusão pelos media, sobretudo a televisão. Além disso, o registo subpadrão sofre menos pressão por parte do purismo linguístico e da ideologia nacionalista. Segundo, a hipótese da maior recetividade do PB aos estrangeirismos é também confirmada no registo subpadrão, embora apenas em relação ao vocabulário de futebol. 


\section{[5.2] Estrangeirismos, heterogeneidade onomasiológica e variação pluricêntrica}

Vamos agora verificar o impacto dos estrangeirismos na heterogeneidade onomasiológica dos conceitos de futebol e de vestuário analisados e na relação diacrónica entre as duas variedades nacionais do português. A análise que se segue é sumária, pretendendo apenas ilustrar a possibilidade de medir o impacto onomasiológico dos estrangeirismos bem como a sua influência na relação entre variedades linguísticas. A análise desenvolvida do impacto dos estrangeirismos na convergência e divergência entre PE e PB encontra-se em Soares da Silva (2014a).

Com base nos cálculos socioletométricos de uniformidade interna (I/I') e de uniformidade externa (U/U'), referidos na secção [3], pudemos encontrar três resultados principais da análise diacrónica das relações entre PE e PB nos últimos 60 anos. A análise desenvolvida destes resultados encontra-se em Soares da Silva (2010).

Primeiro, os cálculos de uniformidade externa (U/U') entre PE e PB indicam divergência clara no corpus do vestuário (uniformidade decrescente) e convergência restringida no corpus do futebol (uniformidade crescente). Relativamente aos termos de futebol, as percentagens de U' mostram convergência de 50 para 70 (U' aumenta 11,39\%) e estabilidade a partir de então, mas as de U não indicam qualquer alteração significativa. Ao mesmo tempo, os números sugerem uma grande distância entre PE e PB nos três períodos: U' 43,78\%/U 35,13\% (anos 50), U' 55,17\%/U 35,90\% (anos 70) e U' 56,76\%/U 36,80\% (anos 2000). Quanto aos termos de vestuário, as percentagens de U' e U evidenciam divergência ao longo do tempo: U' 78,80\%/U 61,65\% (anos 50), U' 65,92\%/U 58,66\% (anos 70) e U' 57,11\%/U $51,44 \%$ (anos 2000), donde U' diminui $12,88 \%$ de 50 para 70 e $8,81 \%$ de 70 para 2000. A hipótese da divergência entre PE e PB é pois confirmada no campo lexical do vestuário. Os termos de vestuário são mais representativos do vocabulário comum e, por isso, os resultados do vestuário estarão mais próximos da realidade sociolinguística. A ligeira convergência no campo do futebol será um efeito da globalização e da estandardização do vocabulário do futebol.

Segundo, comparando as percentagens de uniformidade externa (U/U') entre PE e PB nos três diferentes períodos, conclui-se que (i) no corpus de futebol há mais mudanças do lado do PB, o que sugere que a evolução interna é mais forte e mais rápida no $\mathrm{PB}$ do que no $\mathrm{PE}$; e (ii) no corpus de vestuário as tendências evolutivas das duas variedades são idênticas, o que mostra que a divergência se dá dos dois lados. Tudo isto sugere que não há uma orientação de uma variedade em relação à outra.

Terceiro, os cálculos de uniformidade interna (I/I') indicam maiores mudanças no PB em ambos os campos lexicais. No corpus de futebol, verifica-se um grande aumento de homogeneidade onomasiológica interna no $\mathrm{PB}$, sobretudo de 50 para 70 (de I' $30,85 \%$ para I' $61,85 \%$ ), ao passo que as alterações no PE são menores (de I' 51,86\% para I' 55,63\%). É o PB quem apresenta uma maior diminuição 
no número de termos alternativos. Duas razões poderão explicar este aumento de homogeneidade onomasiológica no PB: (i) grande número de estrangeirismos conservando a forma original em PB50 e sua adaptação a partir dos anos 70; (ii) grande popularidade do futebol no Brasil e o seu prestígio internacional a partir dos anos 60, em virtude da conquista de duas Taças Mundiais consecutivas (1958 e 1962). No corpus do vestuário, as percentagens de uniformidade interna mostram que a homogeneidade onomasiológica diminui de 50 (PE: I' 66,47\%; PB: I' 76,48\%) para 70 (PE: I' 62,99\%; PB: I' 55,71\%) e aumenta de 70 para 2000 (PE: I' 68,75\%; PB: I' $67,87 \%$ ) e que as mudanças são maiores no PB do que no PE.

Podemos então concluir que os estrangeirismos contribuem, até certo ponto, para os resultados principais da relação diacrónica entre as duas variedades nacionais. Por um lado, os empréstimos do inglês no futebol, mais frequentes no PB do que no PE, contribuem para uma distância maior entre as duas variedades nos anos 50 e para uma ligeira convergência entre 50 e 70. Os empréstimos do inglês e do francês no vestuário, em proporções idênticas nas duas variedades e com um ligeiro predomínio no PB, contribuem para a divergência entre PE e PB. Por outro lado, os estrangeirismos de futebol e de vestuário, geralmente mais influentes e mais rapidamente adaptados no $\mathrm{PB}$, contribuem para maiores mudanças de homo/heterogeneidade no PB do que no PE. Obviamente que a influência dos estrangeirismos não é o único fator determinante; outros fatores destes resultados diacrónicos são a endo/exogenidade, os arcaísmos/neologismos e a influência brasileira (ver Soares da Silva 2010).

\section{[6] CONCLUSÕES}

A análise sociocognitiva e socioletométrica baseada num vasto corpus e em perfis onomasiológicos de conceitos de futebol e de vestuário sobre o uso de estrangeirismos nas duas variedades nacionais do português nos últimos 60 anos permitiu verificar que, embora ambas as variedades sejam recetivas aos estrangeirismos, a influência do inglês e de outras línguas estrangeiras é maior no PB do que no PE. A maior recetividade do $\mathrm{PB}$ ao empréstimo lexical manifesta-se tanto no uso de termos estrangeiros diretamente importados como na sua adaptação. Vimos que o $\mathrm{PB}$, tanto no registo formal como no registo informal, importa um número maior de estrangeirismos e adapta-os e integra-os mais frequente e rapidamente do que o PE. Esta maior influência estrangeira no PB, particularmente a dos anglicismos, é mais evidente no vocabulário do futebol, mas no vocabulário do vestuário, com proporções de estrangeirismos idênticas nas duas variedades, não deixa de ser significativo o facto de a influência do francês não ser menor no PB em comparação com o PE. Estes resultados não só confirmam a hipótese de que o PB tende a ser mais recetivo aos estrangeirismos do que o $\mathrm{PE}$, como indiciam quer a emergência quer o tremendo fracasso da atitude purista de defesa da pureza da língua e de guerra contra os estrangeirismos, sobretudo no Brasil (Faraco 2001). 
O uso de estrangeirismos contribuiu para uma maior variação onomasiológica e, assim, para a heterogeneidade onomasiológica dentro e entre PE e PB. Por um lado, os estrangeirismos contribuíram para uma diminuição da uniformidade lexical entre as duas variedades. No caso do PB, o maior uso de estrangeirismos é um dos fatores da sua maior mutabilidade de homo/heterogeneidade lexical ao longo do tempo. Por outro lado, os estrangeirismos contribuíram, até certo ponto, para a divergência clara entre as duas variedades ao longo dos três períodos temporais estudados no vocabulário do vestuário e para a ligeira convergência no vocabulário de futebol.

Finalmente, a perspetiva sociocognitiva, o método onomasiológico baseado em sinónimos denotacionais e os métodos socioletométricos baseados em perfis onomasiológicos de conceitos e num vasto corpus permitem medir e comparar o uso, o sucesso e a evolução dos estrangeirismos nas variedades nacionais do português, bem como analisar o sucesso dos estrangeirismos como um fenómeno multifatorial, determinado simultaneamente por fatores conceptuais, estruturais, socioculturais e pragmático-discursivos.

\section{AGRADECIMENTOS}

Este estudo foi financiado pela Fundação para a Ciência e a Tecnologia, como parte do projeto estratégico PEst-OE/FIL/UI0683/2011 do Centro de Estudos Filosóficos e Humanísticos da Universidade Católica Portuguesa. Agradeço aos revisores Rui Sousa Silva e Luís Trigo os seus esclarecedores e estimulantes comentários e sugestões.

\section{ANEXO}

Perfis de futebol

ÁRBITRO: apitador, árbitro, director da partida, juiz, juiz de campo, «ref(eree)», referi, refre

ÁRBITRO AUXILIAR: árbitro auxiliar, árbitro assistente, auxiliar, 2\%/3ำ $4^{\circ}$ árbitro, bandeirinha, fiscal de linha, juiz de linha, «liner»

AVANÇADO: atacante, avançado, avante, dianteiro, «forward», ponta-de-lança

BALIZA: arco, baliza, cidadela, «goal», gol(o), malhas, marco, meta, rede, redes, vala

BOLA: balão, bola, couro(inho), esfera, esférico, pelota

CANTO: canto, chute de canto, «corner», córner, escanteio, esquinado, pontapé de canto, tiro de canto

DEFESA: «(full-)back», beque, bequeira, defensor, defesa, lateral, líbero, zagueiro

EQUIPA: conjunto, formação, eleven, equipa/e, esquadra, esquadrão, grupo, «match», onze, onzena, plantel, quadro, «team», time, turma

EXTREMO: ala, extremo, ponta, ponteiro 
FALTA: carga, falta, «foul», golpe, infra(c)ção, obstru(c)ção, transgressão, violação (das regras)

FINTA: corte, drible(ing), engano, «feint», finta, firula, ginga, lesa, manobra enganadora, simulação

FORA DE JOGO: adiantamento, banheira, deslocação, fora-de-jogo, impedimento, «offside», posição irregular

GOLO: bola, «goal», gol, golo, ponto, tento

GRANDE PENALIDADE: castigo máximo, castigo-mor, falta máxima, grande penalidade, penalidade, penalidade máxima, penálti (pênalti, pénalti), «penalty»

GUARDA-REDES: arqueiro, «goal-keeper», goleiro, golquíper, guarda-meta, guarda-rede, guarda-redes, guarda-vala, guarda-valas, guardião, «keeper», porteiro, quíper, vigia

JOGADA: jogada, lance

JOGO: batalha, choque, combate, competição, confronto, desafio, disputa, duelo, embate, encontro, jogo, justa, luta, «match», partida, peleja, prélio, prova, pugna

MÉDIO: alfe, central, centro-campista, centro-médio, «half», interior, médio, meia, meiocampista, meio-campo, «midfield», trinco, volante

PONTAPÉ LIVRE: chute (in)direto, falta, «free(-kick)», livre (directo, indirecto), pontapé livre, tiro dire(c)to, tiro livre (direto, indireto)

PONTAPÉ: chute, chuto, «kick(-off)», panázio, pelotada, pontapé, quique, «shoot», tiro

TREINADOR: mister, professor, técnico, treinador

Perfis de vestuário

BLUSA F: «blouse», blusa, blusinha, «bustier», camisa, camisa-body, camisão, camiseiro (inho), camiseta/e, (blusa) «chémisier», (blusa) chemisiê

BLUSÃo M/F: «blazer», blêizer, blêiser, blusão, «bluson», camurça, camurcine, camisa esporte, casaco de pele (de ganga, etc.), colete, parka

CALÇAS M/F: calça, calças, pantalona

CALÇAS CURTAS M/F: bermuda(s), calças-capri, calça(s) corsário, calça(s) curta(s), calças 3/4, calções, «cool pants», corsários, «hot pants», «knikers», «pantacourt», «pedal pusher», «short(s)», «short cuts», «short shorts», shortinho, «slack(s)»

CALÇAS JUSTAS F: «fuseau(x)», fusô, «legging(s)»

CAMISA M: blusão, camisa, camisa de gravata, camisa de manga curta, camisa desportiva, camisa esporte(iva), camisa jeans, camisa social, camiseta, camisete, «camisette»

CAMISOLA M/F: blusa, blusão, blusinha, «body», cachemir, camisa, camisa-de-meia, camiseta, camisinha, camisola, camisolinha, «canoutier», canoutiê, malha, malhinha, moleton, «pull», «pullover», pulôver, suéter, «sweat», «sweat shirt», «sweater» 
CASACO F: «blazer», blêizer, blêiser, casaco, casaquinho/a, «manteau», mantô, paletó, «paletot»

CASACO M: «blazer», blêizer, blêiser, casaco, paletó, «paletot»

CASACO CURTO F: bolero, carmona, casa(i)b(v)eque, casaco curto, casaquilha, colete, colete camiseiro , corpete, corpinho, garibáldi, "gilet», manguito, mini, minicasaco, roupinha, «shortie», vasquinha

CASACO CURTO M: casaco curto, colete, espartilho, gibão, «gilet», jaleca, jaleco, jaqueta, véstia

CASACO DE CERIMÓNIA M/F: «black-tie», casaca, casaco cerimónia, fraque, «manteau», mantô, paletó, «paletot», «pelerine», «smo(c)king», sobrecasaca, «tuxedo»

CASACO DE MALHA M/F: cardigã, «cardigan», casaco/casaquinho de malha (de lã, de tricô), «gilet», japona, malha, «twin-set»

CASACO IMPERMEÁVEL M/F: «ciré», «ciré-maxi», «anorak», canadiana, capa, capa de chuva, casaco impermeável, corta-vento, casaco-gabardina, gabardine/a, impermeável, kispo, parka

CASACO QUENTE M/F: abafo, agasalho, balandrau, capote, casacão, casaco comprido, casaco de abafo/abafar, casaco de agasalho, casaco de/em pele, casaco-sobretudo, «duffle-coat», gabão, «gilet», "manteau», mantô, manto, overcoat, paletó, "pardessus», «pelerine», samarra, sobrecasaca, sobretudo, sobreveste, «trench (coat)»

CONJUNTO F: «complet», completo, conjunto, costume, duas-peças, «ensemble», fatinho, fato, saia-casaco, «tailleur», «toilette», toilete, vestido-casaco

FATO M: beca, completo, costume, fato, terno

JAQUETA M/F: casaca, casaco curto, jaleca, jaqueta, «jaquette», jaquetinha, véstia

JEANS M/F: calça(s) de ganga, calça(s) em denim, calça(s) em jeans, ganga, jeans

SAIA F: kilt, maxi (máxi), maxissaia, micro-mini, micro-saia, míni (mini), mini-saia, minissaia, pareô, saia, saia-calça, saia-calção, saião, sainha, saiote

T-SHIRT M/F: camisa, camiseta/e, «camisette», camisola, licra, «singlet», «tee-shirt», «t-shirt»

VESTIDO F: camiseiro, «chemisier», chemisiê, «shirt-dress», traje/o, veste, vestido(inho), vestido-camisa, vestido-camiseiro, vestido-camiseta, vestido-chemiser(ê), (vestido) cai-cai, (vestido) tomara-que-cai

\section{REFERÊNCIAS}

Baldinger, Kurt. 1964. Sémasiologie et onomasiologie. Revue de Linguistique Romane 28. 249-272.

Croft, William. 2001. Radical Construction Grammar. Oxford University Press. 
Croft, William. 2009. Toward a social cognitive linguistics. Em Vyvyan Evans \& Stephany Pourcel (eds.), New Directions in Cognitive Linguistics, 395-420. John Benjamins.

Faraco, Carlos Alberto. 2001. Estrangeirismos. Guerras em torno da língua. Parábola Editorial.

Geeraerts, Dirk. 2010. Lexical variation in space. Em Peter Auer \& Jürgen Schmidt (eds.), Language in Space. An International Handbook of Linguistic Variation, vol. 1, 821-837. De Gruyter.

Geeraerts, Dirk \& Hubert Cuyckens. 2007. The Oxford Handbook of Cognitive Linguistics. Oxford University Press.

Geeraerts, Dirk, Stefan Grondelaers \& Peter Bakema. 1994. The Structure of Lexical Variation. Meaning, naming, and context. De Gruyter.

Geeraerts, Dirk, Stefan Grondelaers \& Dirk Speelman. 1999. Convergentie en Divergentie in de Nederlandse Woordenschat. Meertens Instituut.

Geeraerts, Dirk, Gitte Kristiansen \& Yves Peirsman (eds.). 2010. Advances in Cognitive Sociolinguistics. De Gruyter.

Goldberg, Adele. 1995. Constructions. A Construction Grammar approach to argument structure. The University of Chicago Press.

Haspelmath, Martin. 2008. Loanword typology: Steps toward a systematic crosslinguistic study of lexical borrowability. Em T. Slotz, D. Bakker \& R. Salas Palomo (eds.), Aspects of Language Contact: New theoretical, methodological and empirical findings with special focus on Romancisation processes, 43-62. De Gruyter.

Haspelmath, Martin \& Uri Tadmor. 2009. Loanwords in the World's Languages: A Comparative Handbook. De Gruyter.

Kristiansen, Gitte \& René Dirven (eds.). 2008. Cognitive Sociolinguistics: Language variation, cultural models, social systems. De Gruyter.

Lakoff, George \& Mark Johnson. 1980. Metaphors We Live By. The University of Chicago Press.

Lakoff, George \& Mark Johnson. 1999. Philosophy in the Flesh: The Embodied Mind and its Challenge to Western Thought. Basic Books.

Langacker, Ronald W. 1987. Foundations of Cognitive Grammar: Theoretical Prerequisites, vol. 1. Stanford University Press. 
Matras, Yaron. 2009. Language contact. Cambridge University Press.

Santos, Diana \& Luís Sarmento. 2003. O projecto AC/DC: Acesso a corpora/disponibilização de corpora. Em Amália Mendes \& Tiago Freitas (eds.), Actas do XVIII Encontro Nacional da Associação Portuguesa de Linguística, 705-717.

Schmid, Hans-Jörg. 2007. Entrenchment, salience and basic levels. Em Dirk Geeraerts \& Hubert Cuyckens (eds.), The Oxford Handbook of Cognitive Linguistics, 117-138. Oxford University Press.

Soares da Silva, Augusto. 2006. O Mundo dos Sentidos em Português: Polissemia, Semântica e Cognição. Almedina.

Soares da Silva, Augusto. 2008. O corpus CONDIV e o estudo da convergência e divergência entre variedades do português. Em Luís Costa, Diana Santos \& Nuno Cardoso (eds.), Perspectivas sobre a Linguateca/Actas do Encontro Linguateca: 10 anos, Linguateca. http://www.linguateca.pt/LivroL10/.

Soares da Silva, Augusto. 2009. A Sociolinguística Cognitiva: razões e objecto de uma nova área de investigação linguística. Revista Portuguesa de Humanidades Estudos Linguísticos 13(1). 191-212.

Soares da Silva, Augusto. 2010. Measuring and parameterizing lexical convergence and divergence between European and Brazilian Portuguese. Em Dirk Geeraerts, Gitte Kristiansen \& Yves Peirsman (eds.), Advances in Cognitive Sociolinguistics, 41-83. De Gruyter.

Soares da Silva, Augusto. 2011. Para a abordagem socioletométrica do pluricentrismo do português europeu e brasileiro: dos indicadores lexicais aos construcionais e atitudinais. Em Augusto Soares da Silva, Amadeu Torres \& Miguel Gonçalves (eds.), Variação linguística e dimensões sociocognitivas, 573-592. Aletheia, Faculdade de Filosofia da UCP.

Soares da Silva, Augusto. 2012. Comparing objective and subjective linguistic distances between European and Brazilian Portuguese. Em Monika Reif, Justyna A. Robinson \& Martin Pütz (eds.), Variation in Language and Language Use: Linguistic, socio-cultural and cognitive perspectives, 244-274. Peter Lang.

Soares da Silva, Augusto. 2013. El pluricentrismo del portugués: Aspectos generales y elementos del enfoque sociolectométrico. Em Rudolf Muhr, Carla Amorós Negre, Carmen Fernández Juncal, Klaus Zimmermann, Emilio Prieto \& Natividad Hernández (eds.), Exploring linguistic standards in non-dominant varieties of pluricentric languages / explorando estándares lingüísticos en variedades no dominantes de lenguas pluricéntricas, 79-90. Peter Lang. 
Soares da Silva, Augusto. 2014a. Measuring and comparing the use and success of loanwords in Portugal and Brazil: A corpus-based and concept-based sociolectometrical approach. Em Eline Zenner \& Gitte Kristiansen (eds.), New Perspectives on Lexical Borrowing: Onomasiological, methodological and phraseological innovations, 101-141. De Gruyter.

Soares da Silva, Augusto. 2014b. Pluricentricity: Language Variation and Sociocognitive Dimensions. De Gruyter.

Soares da Silva, Augusto. 2014c. The pluricentricity of Portuguese: A sociolectometrical approach to divergence between European and Brazilian Portuguese. Em Augusto Soares da Silva (ed.), Pluricentricity: Language Variation and Sociocognitive Dimensions, 143-188. De Gruyter.

Speelman, Dirk, Stefan Grondelaers \& Dirk Geeraerts. 2003. Profile-based linguistic uniformity as a generic method for comparing language varieties. Computers and the Humanities 37. 317-337.

Taylor, John R. 1995. Linguistic Categorization. Prototypes in Linguistic Theory. Oxford University Press 2nd edn.

Zenner, Eline. 2013. Cognitive Contact Linguistics. The macro, meso and micro influence of English on Dutch: University of Leuven. Tese de Doutoramento.

Zenner, Eline \& Gitte Kristiansen (eds.). 2014. New Perspectives on Lexical Borrowing: Onomasiological, methodological and phraseological innovations. De Gruyter.

Zenner, Eline, Dirk Speelman \& Dirk Geeraerts. 2012. Cognitive Sociolinguistics meets loanword research: Measuring variation in the success of anglicisms in Dutch. Cognitive Linguistics 23(4). 749-792.

CONTACTOS

Augusto Soares da Silva

Universidade Católica Portuguesa

assilva@braga.ucp.pt 